\title{
Robotic lobectomy for lung cancer: initial experience of a single institution in Korea
}

\author{
Seha Ahn ${ }^{1}$, Jin Yong Jeong ${ }^{1}$, Hyung Woo Kim², Joong Hyun Ahn' ${ }^{2}$ Giyong Noh ${ }^{3}$, Soo Seog Park ${ }^{3}$ \\ ${ }^{1}$ Department of Thoracic and Cardiovascular Surgery, ${ }^{2}$ Department of Internal Medicine, ${ }^{3}$ Department of Anesthesiology, Incheon St. Mary's \\ Hospital, College of Medicine, The Catholic University of Korea, Seoul, Republic of Korea \\ Correspondence to: Jin Yong Jeong, MD, PhD. Department of Thoracic and Cardiovascular Surgery, Incheon St. Mary's Hospital, College of Medicine, \\ The Catholic University of Korea, 56 Dongsu-ro, Bupyeong-gu, Incheon 21431, Republic of Korea. Email: jeong74@catholic.ac.kr.
}

\begin{abstract}
Background: Robotic surgery is known to have several advantages including magnified three-dimensional vision and angulation of the surgical instruments. To evaluate the feasibility and efficiency of robotic lobectomy in the treatment of lung cancer, we analyzed the outcomes of our initial experiences with robotic lobectomy at a single institution in Korea.

Methods: Eighty-seven patients with lung cancer underwent robotic lobectomy (robotic group: 34 patients) and video-assisted thoracic surgery (VATS) lobectomy (VATS group: 53 patients) between 2011 and 2016 at our hospital. The medical records of these patients were retrospectively analyzed.

Results: The operation times of the two groups were significantly different (robotic group, $293 \pm 74 \mathrm{~min}$; VATS group, $201 \pm 62 \mathrm{~min} ; \mathrm{P}<0.01$ ). Intraoperative blood loss occurred more in the robotic group than in the VATS group (robotic group, $403 \pm 197 \mathrm{~mL}$; VATS group, 298 $\pm 188 \mathrm{~mL} ; \mathrm{P}=0.018$ ). The numbers of lymph nodes dissected in the two groups were significantly different (robotic group, 22 \pm 12 ; VATS group, 14 \pm ; $\mathrm{P}<0.01)$. There was no intraoperative mortality in both groups.

Conclusions: Despite the initial difficulties, robotic lobectomy for lung cancer was a safe and feasible procedure with no operative mortality. If operation time and intraoperative blood loss improve as the learning curve progresses, robotic surgery may overcome the limitations of VATS in lung cancer surgery.
\end{abstract}

Keywords: Lung cancer; lobectomy; robotic surgery; minimally invasive surgery (MIS)

Submitted Nov 09, 2018. Accepted for publication Feb 18, 2019.

doi: $10.21037 /$ acs. 2019.02 .08

View this article at: http://dx.doi.org/10.21037/acs.2019.02.08

\section{Introduction}

Lung cancer is expected to remain a leading cause of cancer-related death in both males and females in the Republic of Korea in 2018 (1). Minimally invasive surgery (MIS) has been performed for lung cancer with a number of advantages compared with the thoracotomy surgery $(2,3)$. Video-assisted thoracic surgery (VATS) lobectomy for lung cancer was first presented in 1992 and has since been adopted worldwide (4-6). However, VATS is subject to a number of limitations, including lack of articulation of the instrument, two-dimensional visualization, and the counterintuitive movement of the instrument (2).

Robotic lobectomy received US Food and Drug
Administration approval in 2000 and was first used for lung cancer in 2003. Since then, the numbers of robotic lobectomies and the numbers of centers performing robotic lobectomy have dramatically increased (7-10). Robotic surgery has several advantages over alternative techniques, including three-dimensional, magnified visualization, the use of an articulating instrument, scaled motion, hand tremor damping and intuitive movement (7). However, the operator has no tactile or force feedback while controlling the robot and performs the surgery based on visual information alone (11).

The benefit of robotic lobectomy compared with VATS lobectomy in the treatment of lung cancer has not been well defined until now. Some studies reported that robotic 


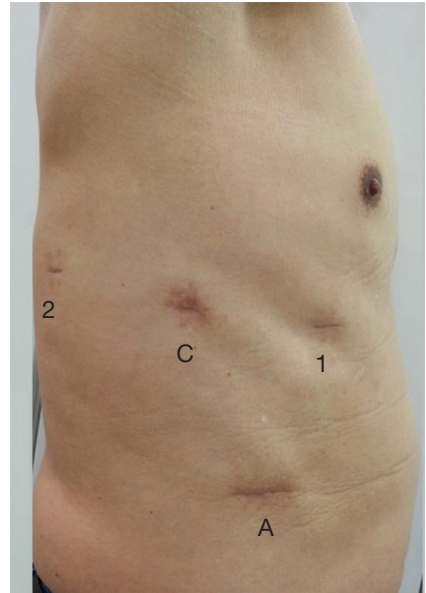

Figure 1 Operation wound after robotic right lobectomy. A camera port incision $(C)$ was placed between the arm 1 and 2 ports (1 and 2). A port incision for arm 3 was placed between the tip of the scapula and the spine; this incision is not shown here. An access assistant incision (A) was then placed to form a triangle with the camera port and arm 1 port.

lobectomy was associated with a longer operative times and a higher rate of intraoperative injury and bleeding than was VATS lobectomy $(9,12)$. However, other studies reported similar perioperative outcomes between robotic and VATS lobectomies, fewer conversions to open procedures and improved mediastinal lymph node dissection (MLND) in robotic lobectomy $(2,13,14)$. To evaluate the feasibility and efficiency of robotic lobectomy in lung cancer surgery, we analyzed the results of our initial experience with robotic lobectomy at a single institution in Korea.

\section{Methods}

Robotic lobectomies were performed between November 2011 and February 2016 on 35 patients with lung lesions. We excluded one patient with a benign lesion and included 34 patients with lung cancer in the robotic lobectomy group (robotic group). During the same period, 53 patients with lung cancer underwent VATS lobectomy by the same surgeon (VATS group). We reviewed the medical records of these patients and analyzed age, sex, pathologic diagnosis, tumor location and size, primary lung cancer stage and operation-related data. Operation time was defined as the duration from the first skin incision to wound closure, including wedge resection for frozen sections to confirm diagnosis when it could not be decided prior to surgery.
When comparing the operation times of the two groups, we excluded all patients who underwent sleeve lobectomy, additional segmentectomy of the other lung lobe, wedge resection of the contralateral lung, or who needed massive bleeding control. When comparing intraoperative blood loss between the two groups, we excluded all patients for whom unexpected massive bleeding needed to be controlled. Dissected lymph nodes were counted in primary lung cancer cases, but not in metastatic cancer cases. When comparing the postoperative hospital stays, we excluded three patients in the VATS group; two patients who were transferred to the medical department due to other medical problems and one patient who died of myocardial infarct.

Written informed consent for robotic and VATS lobectomy was obtained from all patients before any operations were carried out. The authors declare that they have no competing interests. The study was approved by the Institutional Review Board of our medical institution (IRB approval number: OC16RISI0065).

\section{Robotic lobectomy}

Robotic lobectomy was performed with a four-arm technique using the da Vinci surgical system (Intuitive, Sunnyvale, CA, USA) as described by Cerfolio: arm 1 and arm 2 (8-mm ports) in the $6^{\text {th }}$ intercostal space, arm 3 (3-mm port) in the $6^{\text {th }}$ or $7^{\text {th }}$ intercostal space, and a camera port $(12 \mathrm{~mm}$ ) between the arm 1 and 2 ports (15). As in the previous article (16), we made an access assistant incision $(3-5 \mathrm{~cm})$ in the $8^{\text {th }}$ or $9^{\text {th }}$ intercostal space to form a triangle with the camera port and arm 1 port, instead of an assistant port (Figure 1).

\section{VATS lobectomy}

Most operations were performed via three incisions. Two port incisions $(10 \mathrm{~mm})$ were placed in the $6^{\text {th }}$ or $7^{\text {th }}$ intercostal space along the anterior axillary line and the $8^{\text {th }}$ or $9^{\text {th }}$ intercostal space along the posterior axillary line. These port incisions were used for the camera and thoracoscopic instruments. An access incision $(5-7 \mathrm{~cm})$ was placed around the $5^{\text {th }}$ intercostal space, adjacent to the major fissure, along the anterior or mid-axillary line under thoracoscopic vision.

\section{Statistics}

Statistical analysis was performed using SPSS version 18 


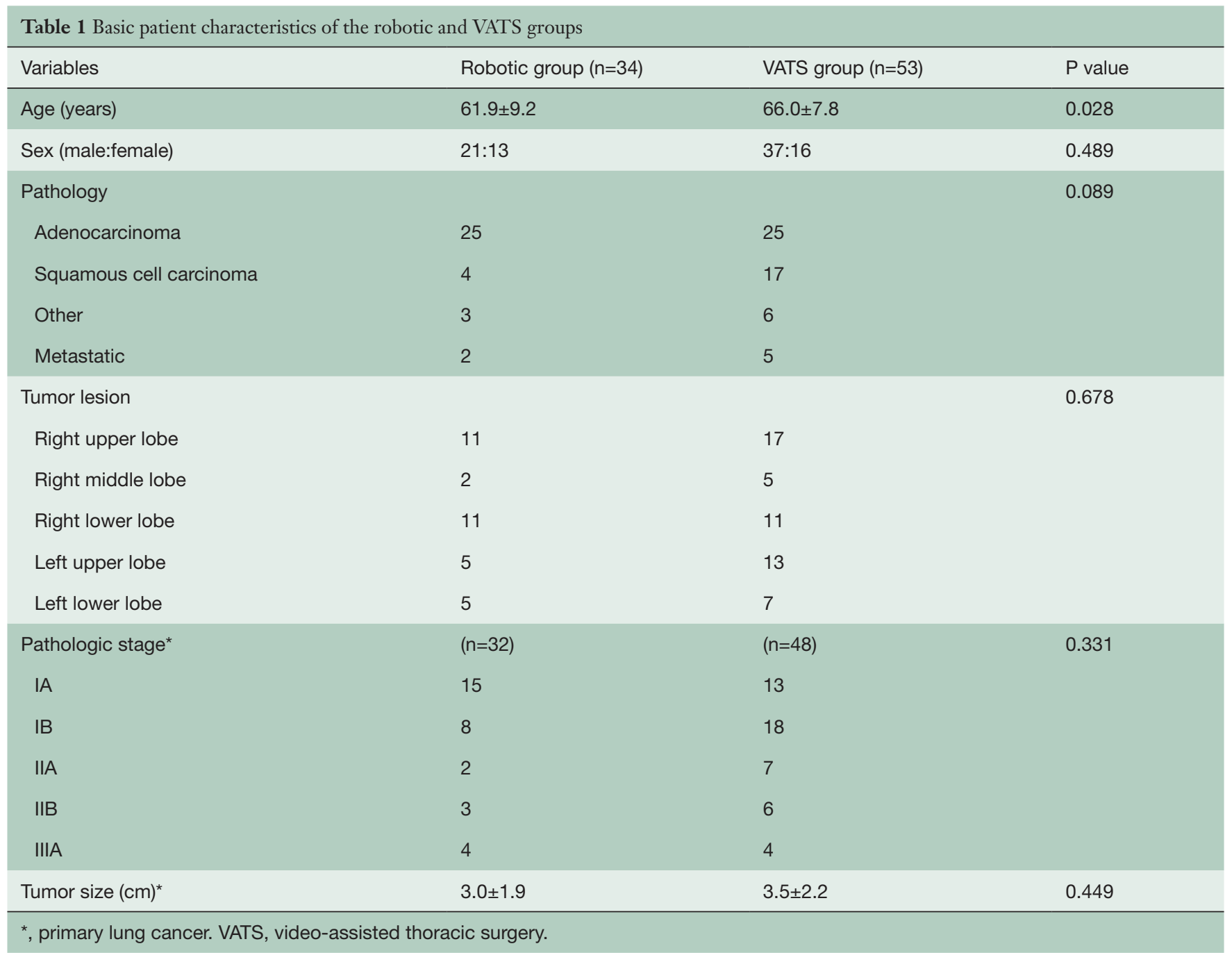

for Windows (SPSS Inc., Chicago, IL, USA). All data are expressed as means \pm standard deviations. The chi-square test was used to compare categorical variables between two groups, whereas the independent $t$-test was used to compare continuous variables. A P value less than 0.05 was considered statistically significant.

\section{Results}

The mean age of the 34 patients ( 21 males, 13 females) in the robotic group was 62 years (range, 44 to 79 years), whereas the mean age of the 53 patients $(37$ males, 16 females) in the VATS group was 66 years (range, 48 to 84 years). The baseline characteristics of the two groups are shown in Table 1 . There were 25 adenocarcinomas and 4 squamous cell carcinomas in the robotic group, and 25 adenocarcinomas and 17 squamous cell carcinomas in the VATS group. The robotic group also included one adenosquamous cell carcinoma, one basaloid carcinoma, and one sarcomatoid carcinoma, whereas the VATS group also included one small cell carcinoma, one large cell carcinoma, one spindle cell carcinoma, one pleomorphic carcinoma, and two neuroendocrine carcinomas. The tumor to be resected was located in the right lung in 24 patients in the robotic group and in 33 patients in the VATS group. The numbers of patients with pathologic stage I, II, and III disease were 23,5 , and 4 in the robotic group and 31 , 13 , and 4 in the VATS group, respectively. There were no significant differences with respect to sex, pathologic type, tumor location, tumor size, or pathologic stage between the 
Table 2 Operation outcomes of robotic and VATS lobectomy

\begin{tabular}{llll}
\hline Variable & Robotic group $(\mathrm{n}=34)$ & VATS group $(\mathrm{n}=53)$ & $\mathrm{P}$ value \\
\hline Operation time (minutes) & $293 \pm 74$ & $201 \pm 62$ & $<0.01$ \\
\hline Intraoperative blood loss $(\mathrm{mL})$ & $403 \pm 197$ & $298 \pm 188$ & 0.018 \\
\hline Conversion to thoracotomy & 1 & 0 & $<.391$ \\
\hline Number of lymph nodes dissected* & $22 \pm 12$ & $14 \pm 7$ & 0.01 \\
\hline Length of postoperative stay (days) & $12 \pm 6$ & $13 \pm 6$ & 0.619 \\
\hline
\end{tabular}

*, primary lung cancer. VATS, video-assisted thoracic surgery.

two groups $(\mathrm{P}>0.05)$. There were no intraoperative deaths in both groups.

In the robotic group, additional wedge resection was performed for contralateral lung lesions in two patients. Unexpected massive bleeding occurred due to vessel injury in three patients, of whom, one underwent direct vessel suture with robotic arms, one underwent prompt vessel division for lobectomy including the lesion with an endostapler, and one underwent thoracotomy conversion. In the VATS group, sleeve lobectomy was performed in four patients, additional wedge resection was performed for a contralateral lung lesion in one patient, and massive bleeding occurred during dissection of diffuse severe pleural adhesions in two patients.

The operation time was significantly longer in the robotic group than in the VATS group (robotic group, $293 \pm 74$ min; VATS group, $201 \pm 62 \mathrm{~min} ; \mathrm{P}<0.01)$. Moreover, significantly more intraoperative blood loss occurred in the robotic group than in the VATS group (robotic group, $403 \pm 197 \mathrm{~mL}$; VATS group, $298 \pm 188 \mathrm{~mL} ; \mathrm{P}=0.018$ ). The mean number of lymph nodes dissected was 22 (range, 5 to 46 ) in the robotic group and 14 (range, 1 to 32) in the VATS group (Table 2); the means of the two groups were significantly different $(\mathrm{P}<0.01)$. However, the length of the postoperative hospital stay was not significantly different between the two groups $(\mathrm{P}>0.05)$.

\section{Discussion}

In 2015, 24,267 new cases of lung cancer are reported to occur in Republic of Korea with more male $(17,015)$ than female $(7,252)$ cases. Moreover, lung cancer led to 17,399 deaths, making lung cancer the leading cause of cancerrelated death in Republic of Korea. The crude mortality rates/100,000 individuals for lung cancer are 49.8, 18.5, and 34.1 in males, females, and both sexes, respectively (1).

MIS has been performed for lung cancer and has several advantages compared with thoracotomy surgery, including smaller surgical incisions, less pain, fewer postoperative complications, shorter hospital stay, quicker recoveries and faster returns to routine daily activity $(2,3)$. However, VATS also has some limitations, including lack of articulation of the instrument, two-dimensional visualization, and counterintuitive movement of the instrument (2). Cuttingedge technological approaches such as robotic surgery have helped overcome some of these limitations. With threedimensional and magnified visualization, the surgeon who controls the surgical instrument in the operator console can perform the operation as if it were a traditional open surgery. Moreover, the articulating robotic instrument has more degrees of motion than the human hand and is superior for performing procedures around and behind certain structures, such as pulmonary vessels and the bronchus. In addition, the articulating robotic instrument is also better at performing procedures within deep and narrow spaces, such as MLND (2). These attributes may facilitate the resection of hilar and mediastinal lymph nodes in robotic surgery compared with VATS (17).

Whether MLND is more beneficial than mediastinal lymph node sampling (MLNS) in the treatment of lung cancer remains controversial. One meta-analysis did not find any significant differences in overall survival, local recurrence rate, or distant metastasis rate between an MLND group and an MLNS group $(18,19)$. Another meta-analysis also found that the 1-year survival rate of an MLND group was similar to that of an MLNS group, but that the 3- and 5-year survival rates were superior in the MLND group (20). On the other hand, other studies have 
supported the idea that MLND is associated with improved survival in early stage and higher stage non-small cell lung cancer $(21,22)$. Lymph node assessment is important for accurate nodal staging of lung cancer. Using MLND, more patients were diagnosed with pathologic N2 disease (17). Moreover, Lee and colleagues showed that although the nodal upstaging rate achieved with robotic surgery was similar to that achieved with VATS, more lymph nodes were harvested with robotic surgery than with VATS (23). Our study demonstrated the same higher nodal harvesting rate for robotic surgery, with this finding reaching significance compared to VATS.

However, certain disadvantages of robotic surgery must be considered. For example, the operator who controls the robotic instruments at the operator console has no tactile or force feedback and must perform the surgery based on visual input alone (11). These limitations may result in various degrees of vascular injury during robotic lobectomy. Most small vascular bleeding episodes can be controlled by compression with sponges; however, if bleeding cannot be controlled, the probability of conversion to thoracotomy is high. The population-based analysis by Paul and colleagues demonstrated a higher rate of iatrogenic bleeding complications and a 5\% emergent thoracotomy conversion rate for bleeding control with robotic lobectomy compared with VATS lobectomy (9). Mahieu and colleagues performed 28 robotic lobectomies, of which two were converted to thoracotomy due to vascular injuries (14). We experienced three cases of vascular injuries that were not adequately controlled by sponge compression. The first case consisted of pulmonary artery injury at the end of vessel dissection; prompt application of the endovascular stapler seized the bleeding in this case. In the second case, the pulmonary artery was injured at the beginning of the dissection; immediate compression with sponges followed by direct closure of the vessel with a robotic suturing instrument controlled the bleeding in this case. In the third case, the apical branch of the pulmonary artery was injured during right upper lobectomy. In this case, compression and direct closure with the robotic instruments failed to control the bleeding, resulting in conversion to thoracotomy and completion of the lobectomy.

Jang and colleagues showed that less intraoperative blood loss occurred in the robotic lobectomy group compared with the initial VATS lobectomy group, whereas the blood loss was not significantly different compared with that of the recent VATS lobectomy group (24). However, we found that more intraoperative blood loss occurred in the robotic lobectomy group compared with the VATS group. Although the operation time in the robotic lobectomy group was not longer than that of the initial VATS lobectomy group, it was longer than that of the recent VATS lobectomy group (24). Similarly, we found that the robotic lobectomy group had a longer operative times compared with the VATS group. However, we had expected that intraoperative blood loss and operation time had the potential to improve dramatically as surgeons progress along the learning curve. We performed robotic lobectomy in 23 patients with lung cancer from March 2016 to June 2018. Their intraoperative blood loss decreased from an average of 403 to $268 \mathrm{~mL}$ compared with the previous one and the operation time decreased from 293 to 243 minutes on average. Robotic lobectomy has been associated with a significantly shorter hospital stay (5.9 days) compared with open thoracotomy (8.2 days), whereas no significant difference was observed with respect to VATS (6.3 days) (25). Similarly, we did not find any significant difference with respect to hospital stay length between the robotic and VATS groups.

In conclusion, our initial experience of robotic lobectomy for lung cancer showed there was no intraoperative mortality. Although robotic surgery was associated with a longer operation time and more intraoperative blood loss than VATS, robotic surgery resulted in more lymph nodes being dissected than VATS. These results suggest that if operation time and intraoperative blood loss improve as the learning curve progresses, robotic surgery may overcome the limitations of VATS with respect to lymph node dissection in lung cancer surgery. However, further evaluation will be needed to determine the extent to which gaining experience in robotic surgery can improve operation time and intraoperative blood loss, in addition to whether the dissection of more lymph nodes actually impacts the long-term survival of patients with lung cancer.

\section{Acknowledgements}

None.

\section{Footnote}

Conflicts of Interest: The authors have no conflicts of interest to declare.

Ethical Statement: The study was approved by the Institutional Review Board of our medical institution (IRB approval 
number: OC16RISI0065).

\section{References}

1. Jung KW, Won YJ, Kong HJ, et al. Cancer Statistics in Korea: Incidence, Mortality, Survival, and Prevalence in 2015. Cancer Res Treat 2018;50:303-16.

2. Velez-Cubian FO, Ng EP, Fontaine JP, et al. RoboticAssisted Videothoracoscopic Surgery of the Lung. Cancer Control 2015;22:314-25.

3. Flores RM, Park BJ, Dycoco J, et al. Lobectomy by video-assisted thoracic surgery (VATS) versus thoracotomy for lung cancer. J Thorac Cardiovasc Surg 2009;138:11-8.

4. Lewis RJ, Caccavale RJ, Sisler GE, et al. Video-assisted thoracic surgical resection of malignant lung tumors. J Thorac Cardiovasc Surg 1992;104:1679-85; discussion $1685-7$.

5. Roviaro G, Rebuffat C, Varoli F, et al. Videoendoscopic pulmonary lobectomy for cancer. Surg Laparosc Endosc 1992;2:244-7.

6. Lee PC, Kamel M, Nasar A, et al. Lobectomy for NonSmall Cell Lung Cancer by Video-Assisted Thoracic Surgery: Effects of Cumulative Institutional Experience on Adequacy of Lymphadenectomy. Ann Thorac Surg 2016;101:1116-22.

7. Ashton RC Jr, Connery CP, Swistel DG, et al. Robot-assisted lobectomy. J Thorac Cardiovasc Surg 2003;126:292-3.

8. Morgan JA, Ginsburg ME, Sonett JR, et al. Thoracoscopic lobectomy using robotic technology. Heart Surg Forum 2003;6:E167-9.

9. Paul S, Jalbert J, Isaacs AJ, et al. Comparative effectiveness of robotic-assisted vs thoracoscopic lobectomy. Chest 2014;146:1505-12.

10. Kang CH, Bok JS, Lee NR, et al. Current Trend of Robotic Thoracic and Cardiovascular Surgeries in Korea: Analysis of Seven-Year National Data. Korean J Thorac Cardiovasc Surg 2015;48:311-7.

11. Benmessaoud C, Kharrazi H, MacDorman KF. Facilitators and barriers to adopting robotic-assisted surgery: contextualizing the unified theory of acceptance and use of technology. PLoS One 2011;6:e16395.

12. Louie BE, Wilson JL, Kim S, et al. Comparison of VideoAssisted Thoracoscopic Surgery and Robotic Approaches for Clinical Stage I and Stage II Non-Small Cell Lung Cancer Using The Society of Thoracic Surgeons Database. Ann Thorac Surg 2016;102:917-24.
13. Mungo B, Hooker CM, Ho JS, et al. Robotic Versus Thoracoscopic Resection for Lung Cancer: Early Results of a New Robotic Program. J Laparoendosc Adv Surg Tech A 2016;26:243-8.

14. Mahieu J, Rinieri P, Bubenheim M, et al. RobotAssisted Thoracoscopic Surgery versus Video-Assisted Thoracoscopic Surgery for Lung Lobectomy: Can a Robotic Approach Improve Short-Term Outcomes and Operative Safety? Thorac Cardiovasc Surg 2016;64:354-62.

15. Cerfolio RJ, Bryant AS, Skylizard L, et al. Initial consecutive experience of completely portal robotic pulmonary resection with 4 arms. J Thorac Cardiovasc Surg 2011;142:740-6.

16. Jo MS, Kim DY, Jeong JY, et al. Robotic sleeve lobectomy with four arms for lung cancer centrally located in the right lower lobe: a case report. J Cardiothorac Surg 2017;12:108.

17. Cerfolio RJ, Bryant AS, Minnich DJ. Complete thoracic mediastinal lymphadenectomy leads to a higher rate of pathologically proven N2 disease in patients with nonsmall cell lung cancer. Ann Thorac Surg 2012;94:902-6.

18. Huang X, Wang J, Chen Q, et al. Mediastinal lymph node dissection versus mediastinal lymph node sampling for early stage non-small cell lung cancer: a systematic review and meta-analysis. PLoS One 2014;9:e109979.

19. Darling GE, Allen MS, Decker PA, et al. Randomized trial of mediastinal lymph node sampling versus complete lymphadenectomy during pulmonary resection in the patient with N0 or N1 (less than hilar) non-small cell carcinoma: results of the American College of Surgery Oncology Group Z0030 Trial. J Thorac Cardiovasc Surg 2011;141:662-70.

20. Dong S, Du J, Li W, et al. Systematic mediastinal lymphadenectomy or mediastinal lymph node sampling in patients with pathological stage I NSCLC: a meta-analysis. World J Surg 2015;39:410-6.

21. Lardinois D, Suter H, Hakki H, et al. Morbidity, survival, and site of recurrence after mediastinal lymph-node dissection versus systematic sampling after complete resection for non-small cell lung cancer. Ann Thorac Surg 2005;80:268-74; discussion 274-5.

22. Keller SM, Adak S, Wagner H, et al. Mediastinal lymph node dissection improves survival in patients with stages II and IIIa non-small cell lung cancer. Eastern Cooperative Oncology Group. Ann Thorac Surg 2000;70:358-65; discussion 365-6.

23. Lee BE, Shapiro M, Rutledge JR, et al. Nodal Upstaging 
in Robotic and Video Assisted Thoracic Surgery

Lobectomy for Clinical N0 Lung Cancer. Ann Thorac

Surg 2015;100:229-33; discussion 233-4.

24. Jang HJ, Lee HS, Park SY, et al. Comparison of the early robot-assisted lobectomy experience to videoassisted thoracic surgery lobectomy for lung cancer: a single-institution case series matching study. Innovations 2011;6:305-10.

25. Kent M, Wang T, Whyte R, et al. Open, video-assisted thoracic surgery, and robotic lobectomy: review of a national database. Ann Thorac Surg 2014;97:236-42; discussion 242-4.

Cite this article as: Ahn S, Jeong JY, Kim HW, Ahn JH, Noh G, Park SS. Robotic lobectomy for lung cancer: initial experience of a single institution in Korea. Ann Cardiothorac Surg 2019;8(2):226-232. doi: 10.21037/acs.2019.02.08 